\title{
ORDER TYPES AND STRUCTURE OF ORDERS
}

\author{
BY \\ ANDRÉ GLEYZAL $\left({ }^{1}\right)$
}

1. Introduction. This paper is concerned with operations on order types or order properties $\alpha$ and the construction of order types related to $\alpha$. The reference throughout is to simply or linearly ordered sets, and we shall speak of $\alpha$ as either property or type. Let $\alpha$ and $\beta$ be any two order types. An order $A$ will be said to be of type $\alpha \beta$ if it is the sum of $\beta$-orders (orders of type $\beta$ ) over an $\alpha$-order; i.e., if $A$ permits of decomposition into nonoverlapping segments each of order type $\beta$, the segments themselves forming an order of type $\alpha$. We have thus associated with every pair of order types $\alpha$ and $\beta$ the product order type $\alpha \beta$.

The definition of product for order types automatically associates with every order type $\alpha$ the order types $\alpha \alpha=\alpha^{2}, \alpha \alpha^{2}=\alpha^{3}, \cdots$. We may furthermore define, for all ordinals $\lambda$, a $\lambda$ th power of $\alpha, \alpha^{\lambda}$, and finally a limit order type $\alpha^{I}$. This order type has certain interesting properties. It has closure with respect to the product operation, for the sum of $\alpha^{I}$-orders over an $\alpha^{I}$-order is an $\alpha^{I}$-order, i.e., $\alpha^{I} \alpha^{I}=\alpha^{I}$. For this reason we call $\alpha^{I}$ iterative. In general, we term an order type $\beta$ having the property that $\beta \beta=\beta$ iterative. $\alpha^{I}$ has the following postulational identification:

1. $\alpha^{I}$ is a supertype of $\alpha$; that is to say, all $\alpha$-orders are $\alpha^{I}$-orders.

2. $\alpha^{I}$ is iterative.

3. $\alpha^{I}$ is minimal in the sense that all iterative supertypes of $\alpha$ are supertypes of $\alpha^{I}$.

It may be shown that these conditions determine a unique $\alpha^{I}$, once $\alpha$ is given. Accordingly, we term $\alpha^{I}$ the minimal iterative supertype of $\alpha$. In particular, when we prescribe $\alpha$ to be the type, "either normal or reverse normal," $\alpha^{I}$, it turns out, is the type scattered $\left({ }^{2}\right)$. Thus we find that scattered orders are constructible from normal and reverse normal orders by the product operation.

Other fundamental operations on orders, such as taking a segment of an order, summing over a normal order, or forming a suborder or superorder of an order, lead to the definition of other order types associated with $\alpha$, and to other properties of order types such as descending, extensive, etc. We denote these associated order types by $\alpha^{D}, \alpha^{E}, \alpha^{F}, \alpha^{R}$, and $\alpha^{N}$. They are unique, de-

Presented to the Society, December 27, 1939, under the title $A$ general theorem on the structure of linear orders; received by the editors January 9, 1940.

(1) I wish to express my appreciation to Professor H. Blumberg for his generous aid in the preparation of this paper. A summary of its principal results is contained in the Proceedings of the National Academy of Sciences, vol. 23 (1937), pp. 291-292.

(2) An order is said to be scattered if it contains no dense suborders. 
pending only upon the choice of $\alpha$, and the first four of them have closure and minimal properties analogous to those described for $\alpha^{I}$. The type $\alpha^{D}$ is shown to be a descending, and $\alpha^{E}$ an extensive order type. Also associated with $\alpha$ are two types which we term $\alpha$-dense and $\alpha$-scattered. They are, as the names indicate, generalizations of dense and scattered. $\alpha$-scattered is iterative and has a certain minimal property with respect to $\alpha$. Of particular interest is the case where $\alpha$ is chosen to be the property of containing $\boldsymbol{N}_{\lambda}$ or more elements, where $\aleph_{\lambda}$ is the $\lambda$ th transfinite cardinal. We denote the two order types associated with this $\alpha$ by $\boldsymbol{\aleph}_{\lambda}$-dense and $\boldsymbol{\aleph}_{\lambda}$-scattered, respectively. $\boldsymbol{\aleph}_{0}$-dense and $\aleph_{0}$-scattered become the properties dense and scattered themselves.

On the basis of these associated order types there may be developed what amounts to an algebra of order types. For example, we may form, by combination, such types as $\alpha^{D E}$ (meaning $\beta^{E}$, where $\beta=\alpha^{D}$ ). The property $\alpha^{D E I}$, important in our considerations, is denoted more simply by $\alpha^{T}$. We find, remarkably, that $\alpha$-scattered is equivalent to $\alpha^{R N D E I}\left(=\alpha^{R N T}\right)$.

A principal result is the following one which gives a decomposition of every order with respect to every order type. It may be stated as follows. If $A$ is any order and $\alpha$ any order type, $A$ is either of type $\alpha^{T}$ or is the sum of $\alpha^{T}$-orders over an order no proper segment $\left({ }^{3}\right)$ of which has type $\alpha^{T}$. This is a generalization of the well known theorem-due to Hausdorff $\left({ }^{4}\right)$ - that every order is either scattered or the sum of scattered orders over a dense order. In this paper, the latter decomposition is the one associated with the property "normal or reverse normal." The order type $\alpha^{T}$, it is found, is simultaneously descending, extensive and iterative. Such a property we term transitive. It may be shown, furthermore, that $\alpha^{T}$ is the minimal transitive supertype of $\alpha$. The property $\alpha$-scattered is transitive for all $\alpha$, and all transitive order types are supertypes of the type scattered. If $\alpha$ is transitive, $\alpha^{T}$ is equivalent to $\alpha$ and the above decomposition theorem implies: If $A$ is any order and $\alpha$ a transitive order type, $A$ is either of type $\alpha$ or the sum of $\alpha$-orders over an order no proper segment of which is of type $\alpha$.

The decompositions we obtain, corresponding to various particular $\alpha$ 's, give insight into the structure of orders and suggest a number of theorems of general nature. One such theorem we prove is that every order of regular $\left(^{5}\right)$ cardinal $\aleph_{\lambda}$ contains either an $\aleph_{\lambda}$-dense order, or the normal order $\omega_{\lambda}$, or the reverse of $\omega_{\lambda}$.

An order $I_{\lambda}$ of transfinite integers is introduced which satisfies the following universality conditions: $I_{\lambda}$ is scattered, of cardinal $\boldsymbol{\aleph}_{\lambda}$, and contains all scattered orders of cardinal less than $\boldsymbol{\aleph}_{\lambda}$.

Problems arise as to properties and methods for constructing orders of

(3) By a proper segment we understand a segment with more than one element.

(4) Grundz üge der Mengenlehre, pp. 95-97.

(5) The cardinal $\boldsymbol{\aleph}_{\lambda}$ and the normal order $\omega_{\lambda}$ initiating the cardinal $\boldsymbol{\aleph}_{\lambda}$ are said to be regular if every suborder of $\omega_{\lambda}$ cofinal with $\omega_{\lambda}$ is of type $\omega_{\lambda}$. 
types such as $\boldsymbol{\aleph}_{\lambda}$-scattered or $\omega_{\lambda}$-scattered. The considerations of this paper lead also to other problems on orders and order types. A number of these are alluded to (see \$12), their solution awaiting future research.

2. Decomposition of an order. Let $A$ be a given order and $\alpha$ an order type or order property. The problem which we wish to consider is that of obtaining a composition of $A$ in terms of orders of type $\alpha$. Later, we give a formal proof of the composition theorem stated in the introduction, but we shall first proceed inductively, tracing step-by-step, the ideas leading to the result we have in mind. To obtain a segmental decomposition, i.e., a separation of the order $A$ into segments, let us begin by associating with an element $a$ of $A$ the elements $e$ of $A$ such that the segment $(a, e)$ or $(e, a)$-taken to include endelements-has property $\alpha$. The elements thus associated with $a$ form a set $S_{a}$ which may or may not be a segment of $A$. To insure that $S_{a}$ form a segment, let us require that $\alpha$ be such that every initial and every final segment of an $\alpha$-order (an order having property $\alpha$ ) be likewise an $\alpha$-order This is equivalent to requiring that every segment of an $\alpha$-order be an $\alpha$-order. This condition upon $\alpha$ we express by saying that $\alpha$ is a descending order property. Furthermore, to insure that two different sets $S_{a}$ have no common elements, we ask that the $\operatorname{sum}\left({ }^{6}\right)$ of two $\alpha$-orders be again an $\alpha$-order. An order property obeying the latter condition we term additive. Therefore, if $\alpha$ is a descending, additive order property, the order $A$ is the sum of nonoverlapping segments $S_{a}$ as defined, and we have determined a composition for $A$. The segments $S_{a}$ themselves form a new order $A_{1}$ if we set $S_{a}<S_{a^{\prime}}$ when $a<a^{\prime}$, and we shall say that $A$ is the sum of $\alpha$-orders over the base $A_{1}$. In the same way as $A$, the order $A_{1}$ may be decomposed with respect to $\alpha$, yielding a new base order $A_{2}$ whose elements are now segments of $A_{1}$. Since each element of $A_{1}$ is a segment of $A$, we may again consider the elements of $A_{2}$ as segments of $A$. Continuing, we secure, for every integer $n$, the base $\operatorname{order} A_{n}$ with elements interpretable as segments of $A$. A "limit" order $A_{\omega}$ may be formed as follows. Let $a$ be an arbitrary element of $A$, and $S_{a}$ the set of elements $e$ of $A$ belonging, for some $n$, to the elements of $A_{n}$ containing $a$. The set $S_{a}$ is a segment of $A$ and the sum of such segments constitutes $A$. Let $A_{\omega}$ be the order with these segments as elements. We may now continue this process beyond the $\omega$ th stage until finally we reach an order $A_{\mu}$, where $\mu$ is a transfinite ordinal, such that no proper segment of $A_{\mu}$ has property $\alpha$. The order $A$ is the sum of segments $S$ of $A$ over the base order $A_{\mu}$. We observe that each segment $S$ may be built up from $\alpha$-orders by means of the following operations:

(1) Forming an order by substituting $\alpha$-orders for the elements of an $\alpha$-order or an order already constructed.

${ }^{(6)}$ By the sum $A+B$ of two orders $A$ and $B$ is meant the order formed by placing all elements of $B$ after all elements of $A$, no change being made in the relative position of elements in $A$ or in $B$. 
(2) Forming an order by substituting $\alpha$-orders or orders already constructed for the elements of a normal or reverse normal order.

Let us call an order which may be built up from $\alpha$-orders by means of these two operations an $\alpha^{T}$-order. We may then say that $A$ is the sum of $\alpha^{T}$-orders over a base order no proper segment of which has property $\alpha$. It may be furthermore shown that $A$ has no proper segment with property $\alpha^{T}$, but we defer the proof until later. We choose to start anew making use of the notions we have just obtained.

3. Iterative order type. Let $\alpha$ and $\beta$ be any two given order properties or types, for example, perfect and scattered. We say that an order is of property $\alpha \beta$ if it is the sum of $\beta$-orders over an $\alpha$-order. As stated in $\S 1$, we term an order property $\alpha$ iterative if it has "closure" with respect to the operation of summing over itself; i.e., if the sum of $\alpha$-orders over an $\alpha$-order is again an $\alpha$-order.

Suppose $\alpha$ is not an iterative order property. We may construct an iterative property $\beta$ implied by $\alpha$, as follows: By $\alpha^{1}$ we understand $\alpha$ itself. Suppose $\alpha^{\mu}$ is defined for all ordinals $\mu$ less than $\lambda$. An order $A$ will be said to have property $\alpha^{\lambda}$ if it is the sum of $\alpha^{\mu}$-orders over an $\alpha$-order, where $\mu<\lambda$ and $\mu$ is permissibly variable. $\beta$ is then the sum type of all types $\left({ }^{7}\right) \alpha^{\lambda}$; i.e., an order $A$ will be said to have property $\beta$ if it has property $\alpha^{\lambda}$ for some $\lambda$. We shall denote the property $\beta$ associated in this way with $\alpha$ by $\alpha^{I}$, the superscript $I$ signifying that $\alpha^{I}$ is iterative, as we show later $\left({ }^{8}\right)$.

We prove, for future reference, that $\alpha^{\mu+\lambda}$ is a supertype of $\alpha^{\lambda} \alpha^{\mu}$. Let us denote by $\alpha^{\bar{\mu}}$ that type which is the sum type of all types $\alpha^{\nu}$, where $\nu<\mu$. Our definition of $\alpha^{\mu}$ may then be written $\alpha^{\mu}=\alpha \alpha^{\bar{\mu}}$. We may then write $\alpha^{\mu+1}=\alpha \alpha^{\overline{\mu+1}}$ $=\alpha\left(\alpha^{\mu}+\alpha^{\bar{\mu}}\right)$. Hence $\alpha^{\mu+1}$ is a supertype of $\alpha \alpha^{\mu}$ and the statement holds for $\lambda=1$. Suppose it holds for all ordinals less than $\lambda$. We may write $\alpha^{\mu+\lambda}=\alpha \alpha^{\overline{\mu+\lambda}}$. Our hypothesis implies, however, that $\alpha^{\overline{\mu+\lambda}}$ is a supertype of $\alpha^{\bar{\lambda}} \alpha^{\mu}$. Therefore $\alpha^{\mu+\lambda}=\alpha \alpha^{\overline{\mu+\lambda}}$ is a supertype of $\alpha \alpha^{\bar{\lambda}} \alpha^{\mu}=\alpha^{\lambda} \alpha^{\mu}\left({ }^{9}\right)$. In particular, we note that $\alpha^{1+\omega}$ is a supertype of $\alpha^{\omega} \alpha=\left(\alpha \alpha^{\bar{\omega}}\right) \alpha=\alpha\left(\alpha^{\bar{\omega}} \alpha\right)=\alpha \alpha^{\bar{\omega}}=\alpha^{\omega}$, as would also be expected from the relation $1+\omega=\omega$. We show that $\alpha^{I}$ has certain minimal and uniqueness properties in relation to $\alpha$, and that these provide a postulational definition for $\alpha^{I}$.

We introduce, for sets in general, a notion of a minimal property. Let $\alpha$ stand for a given set property, and $A$ for a given property of set properties.

${ }^{7}$ ) Let $S$ be a set of order types $\alpha$. By the sum type of the order types of $S$ we understand the order type $\beta$ defined as follows. An order will be said to be of type $\beta$ if it has property $\alpha$ for some $\alpha$ of $S$; otherwise, it will be said not to have type $\beta$.

$\left.{ }^{8}\right)$ It may be true that for a given $\alpha$ there always exists a first ordinal $\lambda$ such that $\alpha^{\lambda}$ is iterative, but the author has no proof of this.

(9) The exponential law $\alpha^{\mu+\lambda}=\alpha^{\lambda} \alpha^{\mu}$ holds if the order consisting of a single element has type $\alpha$. For $\alpha^{\mu}$ is then a supertype of $\alpha^{\nu}$ for all $\nu<\mu$ and, consequently, $\alpha^{\mu+1}=\alpha \alpha^{\overline{\mu+1}}=\alpha \alpha^{\mu}$. Assuming $\alpha^{\mu+\nu}=\alpha^{\nu} \alpha^{\mu}$ for all $\nu<\lambda$, we have $\alpha^{\mu+\lambda}=\alpha \alpha^{\overline{\mu+\lambda}}=\alpha \alpha^{\bar{\lambda}} \alpha^{\mu}=\alpha^{\lambda} \alpha^{\mu}$. 
The set property $\beta$ will be said to be a minimal $A$-property implied by $\alpha$, if it is implied by $\alpha$, has property $A$, and is such that if $\beta^{\prime}$ is any set property implied by $\alpha$ and having property $A$, it is implied by $\beta$. Two minimal $A$-properties implying $\alpha$ are equivalent in the sense that each implies the other. We may thus regard the minimal $A$-property implied by $\alpha$ as uniquely determined-if it exists. We shall therefore speak of "the" instead of "a" minimal property.

THEOREM 1. $\alpha^{I}$ is the minimal iterative property implied by $\alpha$.

Proof. Suppose an order $A$ is a sum of $\alpha^{I}$-orders $A_{\lambda}$ over an $\alpha^{I}$-order $\Lambda=\{\lambda\}$, the subscript $\lambda$ ranging over all the elements of the order $\Lambda$. Each $A_{\lambda}$ is an $\alpha^{\mu}$-order for some ordinal $\mu$. We set $\sigma$ equal to the first ordinal larger than any of the ordinals $\mu$. If $\Lambda$ is an $\alpha^{\nu}$-order, $A$ is an $\alpha^{\nu} \alpha^{\sigma}$-order. Therefore $A$ is an $\alpha^{\sigma+\nu}$-order and hence an $\alpha^{I}$-order. $\alpha^{I}$ is therefore iterative. Now let $\beta$ be an iterative property implied by $\alpha$. Assume $\beta$ is implied by $\alpha^{\mu}$ for $\mu<\lambda$. $\beta$ is then implied by $\alpha^{\lambda}$ since $\beta$ is iterative. Consequently $\beta$ is implied by $\alpha^{\lambda}$ for all ordinals $\lambda$, that is, by $\alpha^{I}$; and the theorem is true.

There is thus uniquely associated with every $\alpha$ the property $\alpha^{I}$ which is the minimal iterative property implied by $\alpha$; i.e., the minimal iterative type which includes $\alpha$ as subtype. We shall say alternatively, that $\alpha^{I}$ is the minimal iterative supertype of $\alpha$. The latter phrasing will also be employed, when convenient, for order type properties other than iterative.

4. Descending order type. We consider now the property descending for order types. Let us denote by $\alpha^{D}$ the property of being a segment of an $\alpha$-order. A segment of an $\alpha^{D}$-order is a segment of an $\alpha$-order and consequently an $\alpha^{D}$-order. Thus $\alpha^{D}$ is descending. If $\beta$ is a descending property implied by $\alpha$, every segment of every $\alpha$-order is a $\beta$-order and $\beta$ is implied by $\alpha^{D}$. Accordingly, we may state

Theorem 2. $\alpha^{D}$ is the minimal descending supertype of $\alpha$.

One may ask the nature of the properties $\alpha^{D I}\left(=\beta^{I}\right.$, where $\left.\beta=\alpha^{D}\right)$, or $\alpha^{I D}$, etc., composed by combining the above described processes. We find that

THEOREM 3. $\alpha^{D I}$ is the minimal descending and iterative supertype of $\alpha$.

Proof. $\alpha^{D}=\left(\alpha^{D}\right)^{1}$ is descending, as we have seen. Suppose $\left(\alpha^{D}\right)^{\mu}$, for ordinals $\mu<\lambda$, is descending. If an order $A$ has property $\left(\alpha^{D}\right)^{\lambda}$, it is the sum of $\left(\alpha^{D}\right)^{\mu}$-orders, $\mu<\lambda$, over an $\alpha^{D}$-order, and every segment $S$ of $A$ is the sum of segments of $\left(\alpha^{D}\right)^{\mu}$-orders over a segment of an $\alpha^{D}$-order. Hence $S$ is the sum of $\left(\alpha^{D}\right)^{\mu}$-orders over an $\alpha^{D}$-order and has property $\left(\alpha^{D}\right)^{\lambda}$. Therefore $\left(\alpha^{D}\right)^{\lambda}$ is descending for all ordinals $\lambda$ and it follows that $\alpha^{D I}$ is descending. By Theorem 1 , $\alpha^{D I}$ is iterative. Suppose now $\beta$ is a descending and iterative property implied by $\alpha$. Then $\beta$ is implied by $\alpha^{D}$, and therefore by $\alpha^{D I}$, for $\alpha^{D}$ and $\alpha^{D I}$ are minimal. Thus $\alpha^{D I}$ is descending and iterative and is minimal, as was to be proved. 
5. Extensive order type. We introduce a third property-again a closure property-corresponding to the operation described above ( $\$ 2)$, of summing orders over normal orders or reverse normal orders. An order type $\alpha$ will be termed extensive if the sum of $\alpha$-orders over a normal order or a reverse normal order is an $\alpha$-order. We prove later that the minimal extensive supertype of $\alpha$ exists for all $\alpha$, and is equivalent to $\sigma \alpha$, where $\sigma$ is the property scattered. In conformity with the notation previously employed for supertypes of $\alpha$, we shall denote $\sigma \alpha$ by $\alpha^{E}$.

6. Transitive order type. The constructions of $\alpha^{D}, \alpha^{I}$ and $\alpha^{E}$ are based on three operations described as follows. $\alpha^{D}$ has closure with respect to the operation of taking segments, for a segment of an $\alpha^{D}$-order is an $\alpha^{D}$-order. Accordingly, we term this operation a $D$-operation. Similarly, $\alpha^{I}$ has closure with respect to the $I$-operation of summing orders of a certain type over orders of the same type. Also, $\alpha^{E}$ has closure with respect to the $E$-operation of summing over normal or reverse normal orders. We now define an order type having closure with respect to all three of the above operations. An order property will be said to be transitive if it is iterative, descending and extensive. Later it is shown that the minimal transitive supertype of $\alpha$ exists and is the order type $\left(\sigma \alpha^{D}\right)^{I}$.

If $\alpha$ is descending, a single element has property $\alpha$. Consequently a descending and extensive order type includes normal and reverse normal orders as subtype. Later, we shall see that a transitive order type includes scattered as subtype.

7. Decomposition of an order into $\alpha$-orders. We prove now the following fundamental decomposition theorem:

ThEOREM 4a. If $\alpha$ is a descending and iterative order property, and $A$ an order whose normal orders and reverse normal orders have property $\alpha$, then $A$ has property $\alpha$ or is the sum of $\alpha$-orders over an order no proper segment of which has property $\alpha$.

Proof. In the special case where $A$ consists of exactly one element, the theorem is true. Suppose $A$ has more than one element. We shall say that a segment of $A$ is a maximal $\alpha$-segment if it has property $\alpha$ and no segment properly containing it has property $\alpha$. Every element $a$ of $A$ is contained in a maximal $\alpha$-segment. For let $S_{a}$ be the set of elements $e$ such that the segment $\langle a, e\rangle$ or $\langle e, a\rangle$ has property $\alpha$, the symbol \langle\rangle signifying that the end points of the segment are included. The set $S_{a}$ is a segment of $A$. For if $e^{\prime}$ is an element of $A$ between $a$ and an element $e$ of $S_{a},\left\langle a, e^{\prime}\right\rangle$ or $\left\langle e^{\prime}, a\right\rangle$ is a segment of $\langle a, e\rangle$ or $\langle e, a\rangle$ respectively, and, since $\alpha$ is descending, has property $\alpha$. Thus $e^{\prime}$ is an element of $S_{a}$. The segment $S_{a}$ has property $\alpha$. For let $a=e_{1}, e_{2}, \cdots ; \cdots, e_{\lambda}, \cdots$ be a normal suborder of $S_{a}$ cofinal with $S_{a}$. A segment $\left(e_{\lambda}, e_{\lambda+1}\right)$-taken to include $e_{\lambda+1}$ but not $e_{\lambda}$-is an $\alpha$-order, since $\left\langle a, e_{\lambda+1}\right\rangle$ is an $\alpha$-order. By hypothesis, the normal order $e_{1}, e_{2}, \cdots ; \cdots, e_{\lambda}, \cdots$ 
is an $\alpha$-order. Thus the suborder of elements of $S_{a}$ to the right of $a$ is the sum of $\alpha$-orders over an $\alpha$-order and therefore has property $\alpha$. Similarly, the suborder of $S_{a}$ to the left of $a$ is an $\alpha$-order. Thus, the segment $S_{a}$ is the sum of at most three $\alpha$-orders and is consequently an $\alpha$-order, since, by hypothesis, every finite suborder of $A$ is an $\alpha$-order. From our definition of $S_{a}$, it follows that no segment properly containing $S_{a}$ has property $\alpha$, and $S_{a}$ is hence a maximal $\alpha$-segment. Moreover, no two distinct maximal $\alpha$-segments have elements in common, for the sum of two such segments forms an $\alpha$-order properly containing each of them, contrary to the definitional property of the maximal $\alpha$-segment. Let $B$ be the order consisting of these maximal $\alpha$-segments. We have shown that $A$ is the sum of maximal $\alpha$-segments over the order $B$. No proper segment of $B$ has property $\alpha$. For suppose there exists such a segment. Then there exists a subsegment $\left\langle S_{a}, S_{b}\right\rangle, S_{a} \neq S_{b}$, of $B$ which has property $\alpha$. Since $\alpha$ is iterative, the set of elements of $A$ composing the segment $\left\langle S_{a}, S_{b}\right\rangle$ has property $\alpha$. It follows that $b$ is an element of $S_{a}$ and consequently that $S_{a}=S_{b}$, contrary to hypothesis. The theorem is thus proved.

THEOREM 4b. If $\alpha$ is a transitive order property and $A$ a given order, then $A$ either has property $\alpha$ or is the sum of $\alpha$-orders over an order no proper segment of which has property $\alpha$.

Proof. We have seen that $\alpha$ includes the order types normal and reverse normal as subtypes. In particular, all normal and reverse normal orders contained by $A$ as suborders are $\alpha$-orders, and Theorem 4a applies.

THEOREM 4c. If $\alpha$ is a transitive order property and $A$ a given order, then $A$ either has property $\alpha$ or is the sum of $\alpha$-orders over a dense order.

Proof. Suppose $A$ is not an $\alpha$-order. Then, by Theorem $4 \mathrm{~b}, A$ is the sum of $\alpha$-orders over an order $B$ no proper segment of which has property $\alpha$. Every proper segment of $B$ consequently does not consist of a finite number of elements, since $\alpha$ includes finite order types. We conclude $B$ is a dense order and the theorem follows.

8. Properties of the type scattered. By means of the above decompositions, we prove a number of theorems concerning the type scattered. For convenience, we shall denote this type by the symbol $\sigma$.

THEOREM 5. The order type scattered is transitive.

Proof. For suppose an order $A$ has property $\sigma$. Then it contains no dense suborders and surely no segment of $A$ contains a dense suborder. Consequently, $\sigma$ is a descending order property. $\sigma$ is iterative; for let $A$ be an order which is the sum of $\sigma$-orders $A_{\lambda}$ over a $\sigma$-order $\Lambda=\{\lambda\}$. Assume there exists a dense suborder $D$ of $A$. No $A_{\lambda}$ contains more than one element of $D$ since otherwise $A_{\lambda}$ would contain a dense order. Thus $D$ is a dense suborder of $\Lambda$, contrary to hypothesis. $A$ therefore is scattered and consequently $\sigma$ is an 
iterative order property. Furthermore, normal and reverse normal orders are subtypes of scattered orders and we conclude that $\sigma$ is transitive. Substituting $\sigma$ for $\alpha$ in the decomposition theorem, there results

THEOREM 6. Every order is either scattered or the sum of scattered orders over a dense order $\left({ }^{10}\right)$.

THEOREM 7. Every transitive order type includes the type scattered as subtype.

Proof. Let $\alpha$ be a transitive order type and $A$ any scattered order. By Theorem $4 \mathrm{c}, A$ has either property $\alpha$ or is the sum of $\alpha$-orders over a dense order. In the latter case $A$ would contain a dense suborder, contrary to hypothesis. Therefore $A$ has property $\alpha$ and the theorem is proved.

9. Minimal supertypes. We now prove the following theorem:

Theorem 8. The minimal iterative order type which includes normal and reverse normal as subtypes is the type scattered.

Proof. Let $\alpha$ be the property of being either a normal or reverse normal order. We have seen (Theorem 3 ) that $\alpha^{D I}$ is descending and iterative. But $\alpha^{D}=\alpha$, and consequently $\alpha^{D I}=\alpha^{I}$. Thus the minimal iterative property $\alpha^{I}$ implied by $\alpha$ exists and is descending. $\alpha^{I}$ is surely extensive since it is iterative and includes normal and reverse normal as subtypes. Therefore $\alpha^{I}$ is transitive and hence contains $\sigma$ as subtype. But, since $\sigma$ is transitive, it is iterative, and must therefore include the minimal type $\alpha^{I}$. Thus $\alpha^{I}$ is equivalent to $\sigma$ and the theorem is valid.

THEOREM 9. If $\alpha$ is any order type, the minimal extensive supertype of $\alpha$ is $\sigma \alpha$, where $\sigma$ is the order type scattered.

Proof. Let $\beta$ be the property normal or reverse normal. Every $\beta \sigma$-order is a $\sigma^{2}$-order, hence a $\sigma$-order. Thus $\beta \sigma \alpha=\sigma \alpha$, and $\sigma \alpha$ is extensive. Let now $\gamma$ be any extensive order type including $\alpha$ as subtype. $\gamma$ then includes $\beta \alpha$, hence includes $\beta \beta \alpha=\beta^{2} \alpha, \beta^{3} \alpha$, etc. Suppose $\gamma$ includes $\beta^{\mu} \alpha$ as subtype for $\mu<\lambda$. It then includes the sum of $\beta^{\mu} \alpha$-orders, $\mu<\lambda$, over a $\beta$-order; that is, $\beta^{\lambda} \alpha$ as subtype. Thus $\gamma$ includes $\beta^{\lambda} \alpha$ for all ordinals $\lambda$. Therefore $\gamma$ includes $\beta^{I} \alpha$ as subtype, and since, by Theorem $8, \beta^{I}=\sigma$, we conclude that $\sigma \alpha$ is minimal as stated in the theorem.

TheOREM 10. If $\alpha$ is any order type, the minimal transitive supertype of $\alpha$ is the type $\left(\sigma \alpha^{D}\right)^{I}=\alpha^{D E I}$.

Proof. In general, the product of two descending order types is a descending order type, and we infer that $\sigma \alpha^{D}$ is descending. By Theorem 3, then, $\left(\sigma \alpha^{D}\right)^{I}$ is descending and iterative. Clearly, $\left(\sigma \alpha^{D}\right)^{I}$ includes $\sigma$, and hence in-

(10) Cf. Hausdorff, loc. cit. 
cludes normal and reverse normal as subtype. Hence, since $\left(\sigma \alpha^{D}\right)^{I}$ is iterative, it is extensive. Consequently, $\left(\sigma \alpha^{D}\right)^{I}$ is transitive. Now, let $\beta$ be any transitive type which includes $\alpha$ as subtype. $\beta$ is descending and includes therefore the minimal property $\alpha^{D}$. Likewise, $\beta$ is extensive and includes therefore $\sigma \alpha^{D}$. Again, since $\beta$ is iterative, it includes $\left(\sigma \alpha^{D}\right)^{I}$. Thus $\left(\sigma \alpha^{D}\right)^{I}$ is the minimal transitive order type called for in the theorem.

We denote, for brevity, the transitive property $\left(\sigma \alpha^{D}\right)^{I}$ associated with $\alpha$, by $\alpha^{T}$. Combining Theorems $4 \mathrm{~b}$ and 10 , we may state

Theorem 11. If $A$ is any order, and $\alpha$ any given order property, either $A$ has property $\alpha^{T}$ or is the sum of $\alpha^{T}$-orders over an order no proper segment of which has property $\alpha^{T}$, where $\alpha^{T}$ is the minimal transitive property implied by $\alpha$.

We now determine minimal properties for a number of particular order properties. Instead of speaking of an order property we will find it convenient, on occasion, to speak of the set of orders having the property. We introduce, for this purpose, the following terminology. Let $S$ be a given set of orders, and $\sigma$ the property of belonging to $S$. We understand by the minimal, iterative set containing $S$ the set $M$ of orders such that the property of belonging to $M$ is equivalent to the minimal iterative property implied by $\sigma$. An analogous phrasing will be used for order type properties other than iterative.

THEOREм 12. The minimal iterative and descending set containing the set which consists of the single element $\omega_{\lambda}$ ( $\omega_{\lambda}$-reversed) is the set of all normal orders (reverse normal orders) of cardinal $\boldsymbol{\aleph}_{\lambda}$ or less.

Proof. Clearly, the set of all normal orders of cardinal $\boldsymbol{\aleph}_{\lambda}$ or less is iterative and descending. Suppose all proper initial segments of $\nu$, where $\nu$ is a normal order of cardinal $\boldsymbol{\aleph}_{\lambda}$ belong to $M$, the minimal iterative and descending set containing $\omega_{\lambda}$. If $\nu$ has a last element, $\nu$ itself is an element of $M$ since the normal orders $\nu-1,1$ and 2 are in $M$. If $\nu$ has no last element, there exists a normal suborder of ordinals $\nu_{1}, \nu_{2}, \cdots ; \nu_{\sigma}, \cdots$ of $\nu$, cofinal with $\nu$ and of type $\omega_{\lambda}$ or less. Thus $\nu$ is an initial segment of the normal order whose ordinal is $\nu_{1}+\nu_{2}+\cdots ;+\cdots+\nu_{\sigma}+\cdots$, with $\nu_{\sigma}$ in $M$. Consequently $\nu$ is an element of $M$-similarly for reverse normal orders.

THEOREM 13. The minimal iterative and descending set containing the set consisting of the two elements $\omega_{\lambda}, \omega_{\lambda}$-reversed, is the set of all scattered orders of cardinal $\boldsymbol{\aleph}_{\lambda}$ or less.

Proof. Clearly, the set of scattered orders of cardinal $\boldsymbol{\aleph}_{\lambda}$ or less is iterative and descending. Now, let $\alpha$ be any iterative and descending order type which includes the type $\omega_{\lambda}$ and $\omega_{\lambda}$-reversed, and let $A$ be any scattered order of cardinal $\aleph_{\lambda}$ or less. By Theorem 12, all normal orders and reverse normal orders of $A$ have property $\alpha$. But by Theorem $4 \mathrm{a}, A$ is either an $\alpha$-order or is the sum of $\alpha$-orders over a dense order. In the latter case $A$ would contain a 
dense order, contrary to hypothesis. Thus the scattered orders of cardinal $\boldsymbol{\aleph}_{\lambda}$ have property $\alpha$ and constitute the minimal set described in the theorem.

We choose, thirdly, for a particular set of orders, the set $S$ of orders of cardinal less than $\aleph_{\lambda}$. We have seen that by means of the operations of taking segments and summing over certain orders, we may construct the orders of the set $M$ which is the minimal transitive set containing $S$ as subset. We inquire now as to the nature of an order of $M$. The decomposition theorem shows us that an order $A$ is either an order of $M$ or is the sum of orders belonging to $M$ over an order $B$ no proper segment of which belongs to $M$. Suppose the latter is true. Then no proper segment of $B$ is of cardinal less than $\boldsymbol{\aleph}_{\lambda}$ and consequently every proper segment of $B$ is of cardinal $\boldsymbol{N}_{\lambda}$ or more. This property of $B$ suggests the notion $\boldsymbol{\aleph}_{\lambda}$-dense which we define as follows: An order will be said to be $\boldsymbol{\aleph}_{\lambda}$-dense if it has more than one element and every proper segment of it contains $\boldsymbol{\aleph}_{\lambda}$ or more elements. Thus $\boldsymbol{\aleph}_{\lambda}$-dense is a generalization of the property dense, $\boldsymbol{\aleph}_{0}$-dense being equivalent to the property dense.

Let us return to the consideration of the properties of an order of $M$. We have found that $A$ is either in $M$ or contains an $\boldsymbol{\aleph}_{\lambda}$-dense suborder. For the purpose of insuring that $A$ be in $M$ we need merely specify that no suborder of $A$ be $\boldsymbol{\aleph}_{\lambda}$-dense. $A$ will then be said to be $\boldsymbol{\aleph}_{\lambda}$-scattered, and in general an order possessing no $\boldsymbol{\aleph}_{\lambda}$-dense suborder will be termed $\boldsymbol{\aleph}_{\lambda}$-scattered. Thus $\boldsymbol{\aleph}_{\lambda}$-scattered is a generalization of the property scattered, the latter being equivalent to $\aleph_{0}$-scattered. Making the guess that, conversely, all orders of $M$ are $\boldsymbol{\aleph}_{\lambda}$-scattered we venture

THEOREM 14. The minimal transitive set which contains the set of all orders of cardinal $\boldsymbol{\aleph}_{\lambda}$ is the set of $\boldsymbol{\aleph}_{\lambda}$-scattered orders.

Proof. We have seen that the set $A$ of $\boldsymbol{\aleph}_{\lambda}$-scattered orders is a subset of $M$, the minimal transitive set containing all orders of cardinal less than $\boldsymbol{N}_{\lambda}$. We show conversely, that $M$ is a subset of $A$. By Theorem 5 , the set of $\boldsymbol{\aleph}_{0}$-scattered orders is a transitive set. If we substitute $\boldsymbol{\aleph}_{\lambda}$-scattered for scattered and $\boldsymbol{\aleph}_{\lambda}$-dense for dense in the proof of this theorem we secure a proof that the property $\boldsymbol{\aleph}_{\lambda}$-scattered is transitive for all $\lambda$. Also, $A$ contains all orders of cardinal less than $\boldsymbol{\aleph}_{\lambda}$. But $M$, being a minimal transitive set, is then a subset of the transitive set $A$. Thus $M$ and $A$ are identical and $\boldsymbol{\aleph}_{\lambda}$-scattered is the required minimal property.

10. Transfinite integer. We now define an order $I$ which is a generalization of the order of the positive and negative integers, and which is a universal scattered order in the sense that it contains all scattered orders as suborders. By transfinite integer we understand the form:

$$
\sum_{i=1}^{n} a_{i} \omega^{\alpha_{i}}=a_{1} \omega^{\alpha_{1}}+a_{2} \omega^{\alpha_{2}}+\cdots+a_{n} \omega^{\alpha_{n}}
$$


where the coefficients $a$ are ordinary integers, and the exponents $\alpha_{i}$ are ordinals decreasing as $i$ increases. We denote the totality of transfinite integers by $I\left({ }^{11}\right)$. An element $\sum_{i=1}^{n} a_{i} \omega^{\alpha_{i}}$ of $I$ will be said to be less than an element $\sum_{i=1}^{m} b_{i} \omega^{\beta_{i}}$ of $I$, if for the first index at which the two forms disagree either $\alpha_{i}<\beta_{i}$ or $\alpha_{i}=\beta_{i}$ and $a_{i}<b_{i}$. It is seen that $I$ thus becomes a linear order. We define sum and product for two transfinite integers in the customary algebraic manner.

THEOREM 15a. I is scattered and every scattered order is similar to a suborder of $I$.

Proof. Let $\alpha$ be the property of being a suborder of $I$ which is not coinitial nor cofinal with $I$. Clearly, $\alpha$ is descending. It is also iterative. For suppose $A$ is the sum of $\alpha$-orders $A_{\lambda}$ over an $\alpha$-order $\Lambda=\{\lambda\} . A$ is then isomorphic with the linear order which consists of the pairs $\left(\lambda, a_{\lambda}\right)$, where $\lambda$ is any element of $\Lambda$ and $a$ any element of $A_{\lambda}$, ordered first according to $\lambda$ and then according to $a_{\lambda}$. Let $\omega^{\alpha}$ be the first power of $\omega$ such that it is greater than, and $-\omega^{\alpha}$ less than, every transfinite integer occurring in the orders $A_{\lambda}$. The transfinite integers of the form

$$
\sum_{j=1}^{m} 2 b_{\lambda j} \omega^{\alpha+\beta_{\lambda j}}+\sum_{i=1}^{n} a_{\lambda i} \omega^{\alpha} \lambda i
$$

where $\sum_{j=1}^{m} b_{\lambda j} \omega^{\beta_{\lambda j}}$ is an element $\lambda$ of $\Lambda$, and $\sum_{i=1}^{n} a_{\lambda_{i}} \omega^{\alpha \lambda t}$ is an element $a_{\lambda}$ of $A_{\lambda}$, constitute a suborder of $I$ similar to $A$, for the correspondence

$$
\sum_{j=1}^{m} 2 b_{\lambda j} \omega^{\alpha+\beta_{\lambda j}}+\sum_{i=1}^{n} a_{\lambda i} \omega^{\alpha} \lambda i \sim\left(\lambda, a_{\lambda}\right)
$$

is biunique and preserves order. $\alpha$ is thus descending and iterative. Suppose now $A$ is a scattered order. It is either an $\alpha$-order or the sum of $\alpha$-orders over a dense order, by Theorem 4c. The latter case cannot occur, for $A$ would then contain a dense order, contrary to hypothesis. $A$ is therefore a suborder of $I$.

We now show, conversely, that every suborder of $I$ is scattered. Upon "writing out" any segment of the order of the transfinite integers, as for example: $1,2,3, \cdots ; \ldots, \omega-3, \omega-2, \omega-1, \omega, \omega+1, \omega+2, \omega+3, \cdots$; $\cdots, 2 \omega-3,2 \omega-2,2 \omega-1,2 \omega, 2 \omega+1,2 \omega+2,2 \omega+3, \cdots ; \cdots, 3 \omega \pm n, \cdots$; $\cdots, \omega^{2} \pm n \omega \pm m, \cdots$, we see that it is locally symmetric in the sense that for every Dedekind cut $(A, B)$ of $I, A$ and $B$ both non-null, there exist subsegments $A_{1}$ of $A$ and $B_{1}$ of $B$, cofinal and coinitial respectively with $A$ and $B$, such that $A_{1}$ is similar to $B_{1}$ reversed( $\left.{ }^{12}\right)$. Suppose $I$ is not a scattered order.

(11) We introduce $I$, rather than a segment of $I$, for convenience, not insisting upon the logical character of $I$ as a totality.

(12) Moreover, it is clear that $I$, or segments of $I$, are the only orders, except for isomorphism, with this local symmetry. 
By Theorem 6, it is the sum of scattered orders $A_{\lambda}$ over a dense order $\Lambda=\{\lambda\}$. No final segment of $A_{\mu}$, where $\mu$ is a fixed element of $\Lambda$, can be similar to the reverse of any initial segment of the set of elements to the right of $A_{\mu}$. For every such segment contains a dense order and $I$ would then not have local symmetry, contrary to fact. We conclude $I$ is scattered.

We shall say that a transfinite integer $\alpha$ is of cardinal $\boldsymbol{\aleph}_{\mu}$ if there are $\boldsymbol{\aleph}_{\mu}$ elements in the segment $(0, \alpha)$. The segment of $I$ comprised of all elements of cardinal less than $\aleph_{\mu}$ we shall indicate by $I_{\mu}$.

THEOREM 15b. $I_{\mu}$ is scattered and contains as suborder all scattered orders of cardinal less than $\boldsymbol{\aleph}_{\mu}$.

Proof. Of course $I_{\mu}$, being a suborder of $I$, is scattered. Let us assume, first, $\boldsymbol{\aleph}_{\mu}$ is a regular cardinal. Substituting $I_{\mu}$ for $I$ in the proof of Theorem 15a there results a proof that the property $\alpha$ of being a suborder of $I_{\mu}$ not coinitial nor cofinal with $I_{\mu}$ is descending and iterative. If $A$ is a scattered order of cardinal less than $\boldsymbol{\aleph}_{\mu}$, all normal and reverse normal suborders of $A$ are of cardinal less than $\boldsymbol{\aleph}_{\mu}$ and hence have property $\alpha$. As above, it follows that $A$ is a suborder of $I_{\mu}$. The theorem is thus proved for regular cardinals $\aleph_{\mu}$. Suppose, now, $\boldsymbol{\aleph}_{\mu}$ is not regular. Then $\boldsymbol{\aleph}_{\mu}$ has no cardinal which is an immediate predecessor and is therefore expressible as a sum of regular cardinals $\aleph_{\nu}$, with $\nu<\mu$. Consequently, if $A$ is a scattered order of cardinal less than $\boldsymbol{\aleph}_{\mu}$, we reason $A$ has cardinal less than some regular cardinal $\aleph_{\nu}$, with $\nu<\mu$. Therefore $A$ is a suborder of $I_{\nu}$. Inasmuch as $I_{\nu}$ is a segment of $I_{\mu}, A$ is a suborder of $I_{\mu}$. The theorem is thus proved for all cardinals $\boldsymbol{\aleph}_{\mu}$.

There are $2 \boldsymbol{\aleph}_{\lambda}$ scattered orders of cardinal $\boldsymbol{\aleph}_{\lambda}$. Accordingly, one may form an order $S$ which is scattered and contains as suborders all scattered orders of cardinal less than $\boldsymbol{\aleph}_{1}$, for example, simply by summing all such orders over a normal order of cardinal $2^{\aleph_{0}}$. Let us compare the two orders $S$ and $I_{1}$, both of which are scattered and contain all scattered orders of cardinal less than $\boldsymbol{\aleph}_{1} . I_{1}$ is certainly of cardinal $\boldsymbol{\aleph}_{1}$ whereas $S$ is of cardinal $2^{\aleph_{0}}$. Hence, they are both of cardinal $\boldsymbol{\aleph}_{1}$, only if the continuum hypothesis $\boldsymbol{\aleph}_{1}=2^{\boldsymbol{N}_{0}}$ is true.

Let us now compare $I_{\mu}$ to the order $S$ composed by summing all scattered orders of cardinal less than $\boldsymbol{\aleph}_{\mu}$ over a normal order. This normal order must be of cardinal $\boldsymbol{\aleph}_{\sigma}=\sum_{\nu<\lambda} 2 \boldsymbol{\aleph}_{\nu}$. Thus $S$ is of cardinal $\boldsymbol{\aleph}_{\sigma}$. If $\boldsymbol{\aleph}_{\mu+1}=2 \boldsymbol{\aleph}_{\mu}$, then $\boldsymbol{\aleph}_{\sigma}=\boldsymbol{\aleph}_{\mu}$ and $S$ is of cardinal $\boldsymbol{\aleph}_{\mu}$. As we have seen, $I_{\mu}$ is of cardinal $\boldsymbol{\aleph}_{\mu}$. We may say, therefore, that our knowledge of whether the cardinal of $S$ is as small as that of $I_{\mu}$ depends upon the validity of the generalized continuum hypothesis.

11. The order types $\alpha$-dense and $\alpha$-scattered. Starting with the order property "has cardinal less than $\boldsymbol{\aleph}_{\lambda}$," we were led to the order properties $\boldsymbol{\aleph}_{\lambda}$-dense and $\boldsymbol{\aleph}_{\lambda}$-scattered. In a similar fashion, starting instead with an arbitrary order property, we are led to the properties we now describe. An order every proper segment of which has more than two elements and contains a suborder of property $\alpha$ will be termed $\alpha$-dense. An order containing no $\alpha$-dense 
suborders will be termed $\alpha$-scattered. If $\alpha$ is the property of containing $\boldsymbol{\aleph}_{\lambda}$ or more elements, $\alpha$-dense and $\alpha$-scattered are equivalent to the previously defined properties $\boldsymbol{\aleph}_{\lambda}$-dense and $\boldsymbol{\aleph}_{\lambda}$-scattered, respectively. Substituting $\alpha$-dense for dense and $\alpha$-scattered for scattered in the proof of Theorem 5 , we may prove

THEOREM 16. If $\alpha$ is any order property, $\alpha$-scattered is transitive.

We note the following alternative wording of the definition of $\alpha$-scattered. An order $A$ is $\alpha$-scattered if every suborder is not $\alpha$-dense. I.e., there exists a proper segment of every suborder which either has exactly two elements or is such that every suborder has property $\alpha^{N}$, where $\alpha^{N}$ denotes the order property: "not of type $\alpha$." In particular, an $\boldsymbol{\aleph}_{0}$-scattered order, that is, a scattered order, may be defined as an order which has the property that every suborder of it contains a segment consisting of exactly two elements. By comparing these definitions of scattered and $\alpha$-scattered, it becomes apparent the type $\alpha$-scattered includes scattered orders as subtype. This would, of course, also be inferred by Theorems 7 and 16 .

Since $\alpha$-scattered is transitive, it furnishes a second decomposition of every order as follows. Every order is either $\alpha$-scattered or the sum of $\alpha$-scattered orders over an order $B$ no proper segment of which has property $\alpha$-scattered. That is to say, every proper segment of $B$, if it exists, contains an $\alpha$-dense order. We conclude that

Theorem 17. If $\alpha$ is any order property and $A$ an order, $A$ is either $\alpha$-scattered or the sum of $\alpha$-scattered orders over an $\alpha$-dense order.

$\alpha$-scattered, being transitive, is equivalent to some property $\beta^{T}$ when $\beta$ is properly chosen. We may ask for an "economical" way to describe $\beta$ in terms of $\alpha$. We have, of course, an "extravagant" solution if we set $\beta$ equal to $\alpha$-scattered. A more "thrifty" answer is developed as follows. We have seen that every suborder of an $\alpha$-scattered order has a proper segment which either consists of exactly two elements or is such that every suborder has property $\alpha^{N}$. Thus $\alpha$-scattered orders contain scattered orders but no $\alpha$-orders. We try

THEOREM 18a. If $\alpha$ is any order property, the order property $\alpha$-scattered is equivalent to the order property $\bar{\alpha}^{T}$, where $\bar{\alpha}$ is the property of containing no suborders of property $\alpha$.

Proof. An $\bar{\alpha}$-order is $\alpha$-scattered since it contains no $\alpha$-orders and hence, surely, no $\alpha$-dense order. Therefore, $\alpha$-scattered is a transitive order type which includes $\bar{\alpha}$-orders as subtype. Since $\bar{\alpha}^{T}$ is the minimal transitive order type which includes $\bar{\alpha}$ as subtype, $\alpha$-scattered includes $\bar{\alpha}^{T}$ as subtype. Suppose, now, $A$ is an $\alpha$-scattered order. By Theorem 11, either $A$ has property $\bar{\alpha}^{T}$ or is the sum of $\bar{\alpha}^{T}$-orders over an order $B$ no proper segment of which has property $\overline{\boldsymbol{\alpha}}^{T}$. In the latter case $B$ has, in particular, no proper segment with 
property $\bar{\alpha}$. Consequently, if $B$ exists, every proper segment of $B$ contains a suborder with property $\alpha$-i.e., contains an $\alpha$-dense suborder. Thus $A$ would contain an $\alpha$-dense suborder, contrary to hypothesis. We conclude that $A$ has property $\bar{\alpha}^{T}$. The latter property is therefore equivalent to the property $\alpha$-scattered.

We determine a construction for $\alpha$-scattered in terms of operations on $\alpha$-orders. Let us consider $\bar{\alpha}$, the order property "containing no $\alpha$-order as suborder." This is equivalent to the property "every suborder has property $\alpha^{N}$." Now, if we denote by $\alpha^{R}$ the property of being a superorder of an $\alpha$-order, the property $\bar{\alpha}$ is equivalent to the property $\alpha^{R N}$. Accordingly, Theorem 18a may be written:

THEOREM 18b. $\alpha$-scattered $=\alpha^{R N D E I}$.

Just as $\alpha^{D D}=\alpha^{D}$, so $\alpha^{R R}=\alpha^{R}$. We shall term $\alpha^{R}$ rising, and, in general, if for an order property $\alpha, \alpha^{R}$ is equivalent to $\alpha$, that is, if every superorder of an $\alpha$-order is an $\alpha$-order, we shall term $\alpha$ rising. It is clear $\alpha^{R}$ is the minimal rising property implied by $\alpha$. On the other hand, the type $\alpha^{R N}$ has the property that a suborder of an $\alpha^{R N}$-order is an $\alpha^{R N}$-order, and we term $\alpha^{R N}$ falling. More generally, we shall name an order property $\alpha$ falling if every suborder of an $\alpha$-order is an $\alpha$-order. If $\alpha$ is any order type we shall denote by $\alpha^{F}$ the property of being a suborder of an $\alpha$-order. Manifestly $\alpha^{F}$ is the minimal falling supertype of $\alpha$. Again we have a "closure equation" $\alpha^{F F}=\alpha^{F}$. We note also, for future reference, that if $\alpha$ is a falling property, $\alpha^{N}$ is a rising property and conversely. Thus $\alpha^{F N}$ is rising and $\alpha^{R N}$ is falling, for all $\alpha$.

In the equation $\alpha$-scattered $=\alpha^{R N D E I}$, we may regard the $R N D E I$-operation as a "solution for $X$ " in the conditional equation $\alpha$-scattered $=\alpha^{X}$. Conversely, we inquire as to possible "solutions" of the equation $\alpha^{X}$-scattered $=\alpha$, where by $X$ we have in mind an operation corresponding to some combination of the letters $F, R, I$, etc. Let us assume there exists a solution. $\alpha^{X}$-scattered is a transitive order type. Clearly, it is also falling. Therefore $\alpha^{F}=\alpha, \alpha^{T}=\alpha$, and $\alpha^{T}=\left(\alpha^{F}\right)^{T}=\alpha^{F T}$. Combining, we obtain $\alpha^{X}$-scattered $=\alpha^{F T}$. But, by Theorem $18 \mathrm{~b}$, we may substitute $\alpha^{X R N D E I}=\alpha^{X R N T}$ for $\alpha^{X}$-scattered. Our conditional equation becomes $\alpha^{X R N T}=\alpha^{F T}$. This is true if $\alpha^{X R N}=\alpha^{F}$, or $\alpha^{X R}=\alpha^{F N}$. The latter equation is equivalent to $\alpha^{X R}=\alpha^{F N R}$, since $\alpha^{F N}$ is rising. Finally, $\alpha^{X R}=\alpha^{F N R}$ is implied by $\alpha^{X}=\alpha^{F N}$. We state

THEOREM 19. If $\alpha$ is any order type, the minimal falling and transitive supertype of $\alpha$ is $\alpha^{F T}=\alpha^{F N}$-scattered.

Proof. We prove the equivalence of $\alpha^{F T}$ and $\alpha^{F N}$-scattered directly. For, since $\alpha^{F N R}=\alpha^{F N}, \alpha^{F N}$-scattered $=\left(\alpha^{F N}\right)^{R N T}=\alpha^{F N R N T}=\alpha^{F N N T}=\alpha^{F T} . \quad \alpha^{F T}$ is minimal for, by Theorem $10, \alpha^{F T}=\left(\sigma \alpha^{F D}\right)^{I}=\left(\sigma \alpha^{F}\right)^{I}$ where $\sigma$ is the order type scattered. The product of two falling order types is, in every case, falling. Thus $\sigma \alpha^{F}$ is falling. In Theorem 3 we proved that $\alpha^{I}$ is descending if $\alpha$ is 
descending. In a similar fashion we may show $\alpha^{I}$ is falling if $\alpha$ is falling. We conclude $\left(\sigma \alpha^{F}\right)^{I}=\alpha^{F T}$ is falling. Furthermore, $\alpha^{F T}$ is transitive since the $T$-operation is performed last. Every falling and transitive order type $\beta$ which includes $\alpha$ includes $\alpha^{F T}$. For $\beta$ includes $\alpha$ implies $\beta$ includes the minimal $\alpha^{F}$. Since $\beta$ is transitive, includes $\alpha^{F}$, and $\alpha^{F T}$ is the minimal transitive type which includes $\alpha^{F}, \beta$ includes $\alpha^{F T}$. Thus $\alpha^{F T}=\alpha^{F N}$-scattered satisfies the requisite minimal condition of the theorem.

We are now in a position to "solve" for $X$ in the equation $\alpha^{X}$-scattered $=\alpha$. There is a solution if and only if $\alpha=\alpha^{F}=\alpha^{F T}$. Then $\alpha^{F N}$-scattered $=\alpha^{F T}=\alpha$; or $\alpha^{N}$-scattered $=\alpha$. Thus $X=N$ is a solution.

We next establish the following characterization for the type $\alpha$-scattered.

THEOREM 20. If $\alpha$ is any order type, the order type $\alpha$-scattered is the minimal falling and transitive supertype of $\alpha^{R N}$.

Proof. The minimal falling and transitive type which includes $\alpha^{R N}$ is, by Theorem 19, $\alpha^{R N F T}$. Since $\alpha^{R N}$ is falling, $\alpha^{R N F}=\alpha^{R N}$ and $\alpha^{R N F T}=\alpha^{R N T}$. But $\alpha^{R N T}=\alpha$-scattered. Thus $\alpha^{R N F T}=\alpha$-scattered, proving the theorem.

The above results show the class of order properties $\alpha$-scattered is identical with the class of order properties which are both transitive and falling. Moreover, a transitive order property is not, in every case, a falling order property. For example, $\alpha^{T}$, where $\alpha$ is the order type of the continuum, does not include the order type of the rational numbers as subtype.

In regard to the notion $\alpha$-dense, it turns out the equation $\alpha^{X}=(\alpha \text {-dense })^{R}$ has a "solution" $\left.{ }^{13}\right)$. For an order is $\alpha$-scattered if no $\alpha$-dense suborders exist. An equivalent statement is that $\alpha$-scattered $=(\alpha \text {-dense })^{R N}$. Thus ( $\alpha$-scattered $)^{N}=\alpha^{R N T N}=(\alpha \text {-dense })^{R}$.

12. Properties of orders with $\aleph_{\lambda}$ elements. The decomposition of Theorem 4 a shows that

THEOREM 21. Every order of cardinal $\boldsymbol{\aleph}_{\lambda}$ containing neither $\omega_{\lambda}$ nor $\omega_{\lambda}-r e-$ iersed, with $\aleph_{\lambda}$ regular, is the sum of orders each of cardinal less than $\aleph_{\lambda}$ over an $\boldsymbol{\aleph}_{\lambda}$-dense order.

Proof. The property $\alpha$ of containing less than $\boldsymbol{\aleph}_{\lambda}$ elements is iterative and descending. With this choice of $\alpha$, Theorem 4 a becomes the above theorem.

Thus every order of cardinal $\boldsymbol{\aleph}_{\lambda}$, containing neither $\omega_{\lambda}$ nor $\omega_{\lambda}$-reversed, with $\boldsymbol{\aleph}_{\lambda}$ regular, contains an $\boldsymbol{\aleph}_{\lambda}$-dense order. It follows that

(13) It seems that in the equation $\alpha^{X}=\alpha$-dense there is no solution for $X$ in terms of the letters $D, E, I, F, R, N$. We may, however, construct an $\alpha$-dense order, once $\alpha$ is given, as follows: Let $A_{1}, A_{2}, \cdots, A_{n}, \cdots$ be a series of $\alpha$-orders. We may form a development $a_{1} a_{2} \cdots a_{n} \cdots$, where the entry $a_{n}$ is an element of $A_{n}$. The set of all such developments, ordered lexicographically, constitutes an $\alpha$-dense order: The set of all such orders determines an order type which might also appropriately be termed an $\omega$ th power of $\alpha$. Cf. $\S 3$. 
THEOREM 22. Every order of regular cardinal $\boldsymbol{\aleph}_{\lambda}$ contains either $\omega_{\lambda}$, or $\omega_{\lambda}$-reversed, or an $\aleph_{\lambda}$-dense order.

Order types $\alpha$ which may serve as a starting point in the formation of new order types and in the study of the structure of orders are: dense, closed, perfect, of cardinal $\aleph_{\lambda}, \omega_{\lambda}, \rho_{\lambda}\left({ }^{14}\right)$, etc. We may then form the order types $\alpha^{D}, \alpha^{E}$, $\alpha^{I}, \alpha^{R}, \alpha^{F}, \alpha^{N}$ and any combination of these to form new order types $\beta$. With each of these $\beta$ 's is associated the transitive types $\beta^{T}$ and $\beta$-scattered each of which provides a segmental decomposition of every order. A number of these associated types and decompositions have been considered in this paper. Others, of possible interest, we leave to future investigation. We note, too, the possibility of introducing order types associated with $\alpha$ by other means such as classification according to properties of Dedekind cuts, properties of initial segments, etc. $\left({ }^{15}\right)$.

(14) See Hausdorff, Mengenlehre, pp. 180-185.

(15) See Hausdorff, Mengenlehre, pp. 142-147.

St. Michael's College,

Winooski Park, Vt. 